\title{
Accurate and Efficient Differentiation Between Normal and Epileptic Seizure of Eyes Using 13 Layer Convolution Neural Network
}

\author{
Veeramosu Priyanka Brahmaiah ${ }^{1 *}$, Yarlagadda Padma Sai ${ }^{1}$, Mahendra N. Giri Prasad $^{2}$ \\ ${ }^{1}$ ECE, VNR Vignana Jyothi Institute of Engineering and Technology, Hyderabad 500090, Telangana, India \\ ${ }^{2}$ ECE, JNTU College of Engineering, Ananthapuramu 515002, Andhra Pradesh, India
}

Corresponding Author Email: priyanka_v@vnrvjiet.in

https://doi.org/10.18280/ts.380427

Received: 15 June 2021

Accepted: 3 August 2021

\section{Keywords:}

background noise, dynamic time wrapping, hidden Markov model, blink features, optimal feature selection, thirteen layer neural network

\begin{abstract}
Epileptic seizure is one which affects the normal brain activities of human being and considered to be a risky disease. The eye ball movement signals pattern plays a significant role in determining the epileptic seizure in precise manner. In addition to it, EOG signals has its influence in detecting epileptic seizure through assessment of eye ball movement signals precisely. Detecting Epilepsy using genetical based Convolutional Neural Network plays a major role in the previous research works. Conversely, the existence of background noise on eye ball signals may impact on the outcome failure. Noise aware Epileptic Seizure Detection using Thirteen Layer Convolution Neural Network (NESD-TLCNN) is adopted in this research to mitigate this issue and thereby ensuring the prediction rate more precisely. Furthermore, Hybrid Dynamic Time Wrapping based Hidden Markov Model (HDWTHMM) is greatly utilized for primary background noise detection and removal by estimating the noise depending on distance metric. Once after the completion of noise estimation, perfect detection of epileptic seizure is accomplished using feature extraction. The peculiar features involved are saccade, fixation and blink features. Subsequently, Particle swarm optimization (PSO) technique is also involved in this research for optimal feature selection. Thirteen Layer Convolution Neural Network (TLCNN) is applied at last for learning and differentiation of epileptic seizure from the normal eyes. This research is being carried out in MATLAB platform which also reveals that the anticipated methodology produces improved outcomes when contrasted with the existing research work.
\end{abstract}

\section{INTRODUCTION}

Epilepsy is considered to be one of the widely recognized neurological illnesses portrayed by epileptic seizures and World Health Organization (WHO) indicates it to be the second crucial neurological ailment apart from stroke [1]. The occurrence of seizures might not depend on circumstances or host attributes [2]. The ill effects are experienced by the epilepsy patients in the course they can't secure themselves and are prone against suffocation, demise, or injury due to due to fainting and traffic accidents [3]. Until this point in time, this ailment is essentially given with prescriptions and medical procedure; there is no complete recovery and no effectual medicines with anticonvulsants for all epilepsy kinds [4].

Electrooculography (EOG) is adopted for identifying epilepsy as the new area of research. Epilepsy in previous research works were detected using EEG signal which is obtained from subjects scalp. The voltage produced in the scalp is taken by using different electrodes positioned on the scalp [5]. An immediate assessment by a doctor is necessitated for recognizing EEG as a significant measure of time and exertion [6]. Moreover, specialists with contrasting degrees of analytic experience here and there report discrepant conclusions on the symptomatic outcomes. In this manner, the advancement of a computerized, PC supported strategy for the analysis of epilepsy is direly required [7].

The potential difference between cornea and retina voltages obtained using electrodes placed on lobes of the subjects [8] is estimated by Electrooculography (EOG) approach. The subsequent sign is known as the electrooculogram and its applications lies in ophthalmological verdict and in recording eye movements. EOG doesn't gauge reaction to individual visual improvements in contrast to the electroretinogram [9]. A sets of terminals are ordinarily positioned either above and underneath the eye or to one side and right of the eye for quantifying eye development. On the off chance that the eye moves from focus position toward one of the two electrodes, this electrodeode "sees" the positive side of the retina and the contrary terminal "sees" the negative side of the retina [10] which leads to a electrodes potential difference. The recorded potential is a proportion of the eye's position, supposing that the resting potential is steady.

EOG signals are greatly utilized for automatic seizure recognition by employing various methodologies [11]. Automatic seizure detection systems' performance mainly relies on EOGs behaviour by means of extracting features. Various researches are carried out for feature extraction and selection techniques [12] and these are carried out in timedomain, frequency-domain or the integration of both the domains. There involve three main challenges in the above domains based approach. The first most challenge is that, very sensitive and robust scarce) to acute discrepancies in seizure patterns which is due to the non-stationary EEG data stationary in addition modifications in its statistical features through 
different subjects and over time for the same subject [13]. The various artifacts such as muscle activities, eye-blinks, and environmental white noise influences on EEG data acquisition systems which is the second case. The seizure detection systems performance accuracy is extremely affected due to the various noise sources which may modify the EEG features.

A Noise aware Epileptic Seizure Detection using Thirteen Layer Convolution Neural Network (NESD-TLCNN) is introduce to mitigate these issues. It also concentrates on prediction and precise determination of discrepancy among the normal eyes and the eyes with epileptic seizure regardless of noise presence. Various procedures are involved in this work for accurate noise concerned epileptic seizure detection.

\section{RELATED WORKS}

Xiahou et al. [14] presented a methodology involving the combination of human eye movement recognition and tracking. The eye elements such as iris and pupils of human eyes are ascertained by means of threshold based eye recognition scheme. The motion feature of eyes comprising translation and velocity play a key role in analysis of eye movement tracking technique. The potential application of eye interaction systems is revealed by designing NUI based application scenarios and real-time tests of different people's eyes recognition and tracking while experimentation.

Kubacki and Jakubowski [15] discussed about the Electroencephalography (EEG), Electrooculography (EOG), and Eye tracking data's theoretically rather than practically. Also presented a review of hybrid BCIs in which interface was assembled with bioactive sensors mounted on the head. The eye signal movements signals by EOG and eye tracking plays a key role in triggering the movement of industrial robot which is turn managed by Ethernet and testing of built interface is accomplished. It was investigated with creating three experiments and people aged 25-35 were utilized.

Cafasso and Karlsson [16] utilized EOG for saccades automatic detection through establishing an algorithm where the combination of Continuous Wavelet Transform Saccade Detection (CWT-SD) and Shape Features are greatly deployed. Whenever assurance for good signal quality can be given, the algorithm will be effective for driving environments. The other aim is exploration of cognitive distraction affecting saccadic rate and amplitude in the course of driving which influences statistically significant reduction in saccadic rate in the course of cognitive load but not in saccade amplitude. The result is validated by future research on bigger datasets and different driving scenarios

Bin Suhaimi et al. [17] exploited a 24-point gazing data for which conversion is done simultaneously for presumption of an imaginary center (i.e., 25th point) on gaze coordinates with 24-point gazing data and transformation to 24-point gazing data is accomplished. To validate the results, conventional method is contrasted with the proposed method.

Baba et al. [18] utilized EOG signal for recognizing eye movement for movement disabilities people communication. This model involves computer, analogue-to-digital converter, digital-to-analogue converter, a direct current (DC) amplifier and a monitor. The eye-gaze position is obtained by target displaying using the monitor. The eye potential derivation and eye-gaze position relation are attained by user looking at the target and at persistent interval target position on the monitor is varied.

Roy et al. [19] established a system for human beings cognitive capabilities understanding by investigating the saccadic movement and its pattern. The movement of the eyeball from one viewpoint to another is referred as saccade. The characterization of sudden deflection of the voltage fluctuation in the EOG is done through the taken saccade action.

Kumar and Sharma [20] introduced a system for virtual reality (VR) games improving the immersive VR experience namely Electrooculogram (EOG) and gaze based hands-free natural interaction system design. VR HMD peripherals are prominent for virtual experience when deployed with conventional interfaces like joysticks, mouse, keyboards, hand-worn data-gloves etc. The see-and-play user interaction in VR games is considered to be a further step. This methodology offers an improvised gaming experience due to the user's virtual environment is intermingling as per the eye movements.

Sharma et al. [21] suggested methodology for eye closing/opening recognition via EOG signals in an online environment. The accuracies outcomes observed are 95.6\% and $91.9 \%$ respectively for 8 healthy subjects for precise detection of eye closing and opening operations. The amalgamation of eye blinks detection leads to $96.9 \%$ improved accuracy.

Schmidt et al. [22] analysed the numerous eye blink detection algorithms performances under various constrictions The electrooculogram- and camera-based blink detection process performance performances are assessed physically and conditionally automated driving phases. The blink detection algorithms performance in both driving modes is utilized for drowsiness impact evaluation by contrasting with alert and drowsy drivers.

Ryu et al. [23] introduced a novelty in eliminating the baseline drift and noise by deploying a differential electrooculography (EOG) signal established on a fixation curve (DOSbFC) and a new electrode positioning structure constructed on eyeglasses. The human-computer interface is managed by implementation of desktop and mobile applications. Experimental EOG eyeglasses and a new detection protocol is acquired by means of proposed method for long-term step-by-step detection of eye movements. This methodology estimates the accumulated EOG signals between the initial eye movement and fixation time differences.

\section{NOISE AWARE EPILEPTIC SEIZURE DETECTION}

An early stage of this study has involved the method, called Hybrid Dynamic Time Wrapping based Hidden Markov Model (HDWT-HMM) to carry out the identification and elimination of Background Noise, in which the Noise has evaluated on the basis of distance parameter. Post-evaluation of Noise, the accuracy in identifying the epileptic seizure has assured through executing the Feature Extraction process. The features, such as Fixation, Blink Features, and Saccade have considered in this study. Subsequently, the implementation of Optimal Feature Selection has done through the strategy, namely Particle Swarm Optimization (PSO). In addition, the approach of Thirteen Layer Convolution Neural Network (TLCNN) has engaged to learn and contrast the epileptic seizure from normal eyes. 


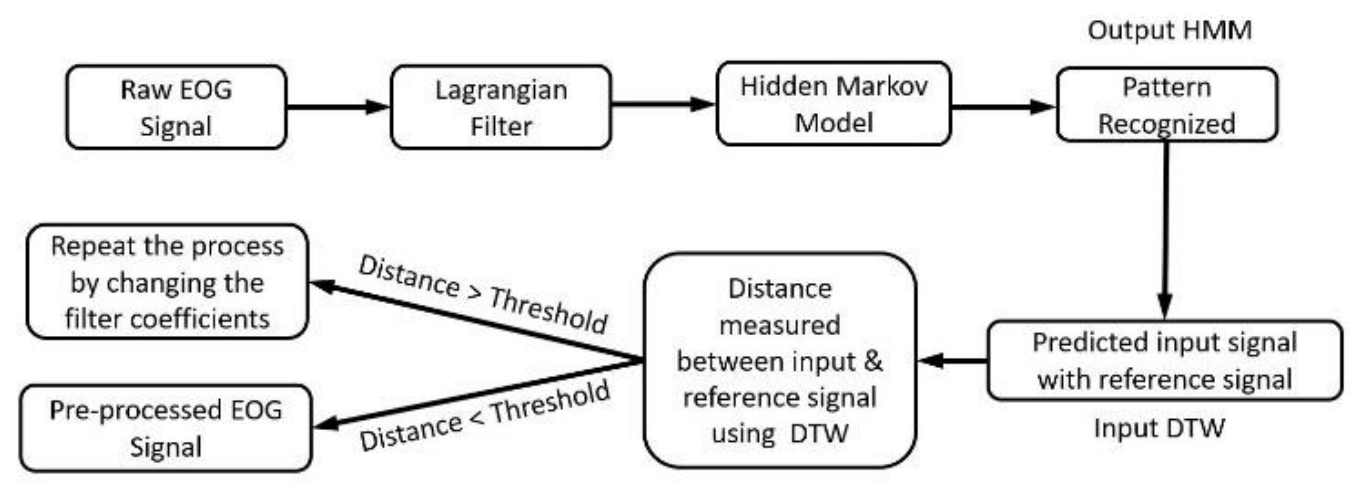

Figure 1. Process flow of HDTWHMM

\subsection{Noise estimation and removal using hybrid dynamic time wrapping based hidden Markov model}

Hidden Markov Model has employed to deal with the estimation of reference patterns, besides the generalization of training data with the reference patterns have done through Dynamic Time Wrapping, which previously determines the distance within recorded speech and a template. Then, the distance of the signals has estimated at every instant that accompanies the warping function. Additionally, the clusters have been trained by HMM that passes within clusters according to the probabilities provided by several models. Eventually, the recoded voice has attained nearly appropriate segmentation by this algorithm. The recoding has processed at noisy places. In some instances, the segmentation process might face the challenge of acquiring the inconsistent values from the algorithm, as it affected by the background noise. Due to the noise interruption, it necessitates increasing the cut off for silence, since it could not expect to be zero. The process of pre-processing using Hybrid Dynamic Time wrapping using HMM is shown in Figure 1.

A method of on-time optimal alignment for the test and the reference patterns has provided by the DTW algorithm in order to furnish the average distance corresponding to the optimal warping path. DTW is known to be a pattern matching algorithm that relies on Bellman's principle of optimality with a non-linear time normalization effect.

The two sequences of the feature vector have aligned with the alignment procedure generated by DTW. i.e. $T_{1}, T_{2}, \ldots, T_{n}$ and $\mathrm{R}_{1}, \mathrm{R}_{2}, \ldots, \mathrm{R}_{\mathrm{n}}$.

Here, a function $\mathrm{f}(\mathrm{i}, \mathrm{j})$ signifies a distance between two feature vectors $\mathrm{Ti}$ and $\mathrm{Ri}$ that has considered as local distance. The local distances have iterated and added them for the optimal predecessor to calculate the global distance. The following equation expresses the optimal global distance:

$$
D(i, j)=\min _{m<=I, k<=j}[D(m, K)]+f(i, j)
$$

In addition, DTW enables the establishment of time scale alignment within two feature vectors, besides it has the capability to procure the long-range dependencies in acoustic data and has the proficiency to attune with the variations in eye movement. The aforementioned factors reveals that the DTW is capable of performing the wide-ranging observation, and HMM is efficient enough to solve the assessment of the detail. So the conclusion can be focused on an amalgamation of DTW and HMM approaches as regards the design of effective framework. The following features can be considered to build the advanced DTW:
The provided input feature vector sequence $T_{1}, T_{2}, \ldots, T_{n}$ and a sequence of states $R_{1}, R_{2}, . ., R_{n}$ (DTW template) can be enforced with the method of DTW. Each states in the advanced DTW has described by the set of parameters.

1. The set of model parameters has represented by $\pi$

2. The estimation of the quality of the model has formulated as,

$$
f(y \mid \pi)=\sum_{q \in Q} f(y, q \mid \pi)
$$

where, the set of overall valid paths has indicated by Q.

3. The quality of path has estimated by,

$$
f(y, q \mid \pi)=f(q \mid \pi) f(y \mid q, \pi)
$$

in which, $f(q \mid \pi)$ signifies a prior measure for traversing through path $\mathrm{q}$, whereas $\mathrm{f}(\mathrm{y}, \mathrm{q} \mid \pi)$ indicates a posterior quality measure.

The Prior path traversal measure has expressed as,

$$
f(q \mid \pi)=\left[\sum_{i=1}^{L(q)} a_{q_{i}}^{W\left(q_{i}\right)}\right]^{p(q)}
$$

Here, $L(q)$ stands for the sequence of the connected segments $\mathrm{q}_{1}, \mathrm{q}_{2}, \ldots, \mathrm{q}_{\mathrm{n}}$ of valid path, while $\mathrm{W}\left(\mathrm{q}_{\mathrm{i}}\right)$ denotes the weight of the ith segment q.

$$
W(q)=\sum_{j=1}^{r_{q}} w_{q} \cdot j
$$

in which, the number of grid points denoted by $r_{\mathrm{q}}$. As a path independent, $\mathrm{p}(\mathrm{q})$ has represented as $\rho$. The estimation of the posterior path quality has defined as the entirety of segment quality that make path.

$$
f(y \mid q, \pi)=\prod_{i=1}^{L(q)} b\left(v_{q i} \mid \pi\right)
$$

Here, the quality measure of the segment $v$ and its respective grid points has symbolized by $b\left(v_{\mathrm{qi}} \mid \pi\right)$.

$$
b\left(v_{q i} \mid \pi\right)=\prod_{j=1}^{L(q)} b_{q}\left(v_{q}(j) \pi\right)
$$

Here, the weighted and the estimation of normalized local similarity for grid point has notated by $b_{q}(\nu \mid \pi)=$ $[p(v \mid \pi)]^{p(q) w_{q}(v)}$, whereas the Gaussian probability density function has represented by $p(v \mid \pi)$. The proposed method is not functioning in a statistic way, as the measurement of path quality might rely on the path and weighting, besides the normalization process disrupt the scenario of statistical feature. 
The forward and backward algorithms has applied to determine the path quality, in which forward variable $\alpha(u)$ has designated as the total of the path quality measure across overall valid path from beginning to ending.

$$
\alpha(u) \triangleq \max _{q \in Q, \text { start }, u}\left\{\prod_{i=1}^{L(q)} a_{q_{i}}^{\rho W\left(q_{i}\right)} \cdot b\left(V_{q_{i}} \mid \pi\right)\right\}
$$

Here, the set of parameters $\pi$ has determined and $V=V^{1}$, $\mathrm{V}^{2}, \ldots, \mathrm{V}^{(\mathrm{n}-1)}, \mathrm{V}^{\mathrm{n}}$ presented as training patterns, with the intention of augmenting $\mathrm{f}(\mathrm{V} / \pi)$ as follows,

$$
\hat{\pi}=\arg \max _{\pi} f(V \mid \pi)
$$

\subsection{Eye feature extraction}

EOG signal characteristics are defined by measuring the variation in amplitude, in volts, with respect to time. The signal has a repetitive pattern for various activities. The development of the classifier and its performance depends on the selection of the right extracted features. Therefore, feature extraction plays a significant role in achieving better accuracy from the classifier.

A feature is defined as a structural, specific or characteristic measurement of the segment, which is extracted from certain pattern. The extracted features can be used to classify the signal into groups by defining their key factors. The process of feature extraction from the EOG signal and optimization of the extracted features are detailed in this section.

It is mandatory to identify the various forms of eye movement to utilize the analysis of epileptic seizure for recognizing the activity. There are three simple kinds of eye movement have identified which has eased by EOG: Saccades, Fixations, and Blinks.

\subsubsection{Saccades}

By nature, the eyes do not being static at the time of seeing an object optically, rather than they keep moving to construct an outline through the appealing portions of that object, only because of the fovea, a tiny pit located in the centre of the retina that enables the vision sharper. The concurrent activities of both eyes has termed as Saccade. The saccadic period relies on their saccade amplitude that is known as the angular distance travelled by the eyes at the time of this activity. The saccadic eye movements usually occur with the amplitude of 20 degrees, and 10 to $100 \mathrm{~ms}$ of duration.

\subsubsection{Fixations}

Fixations refers to the fixed positions of the eyes when the focus in the visual object has retained at a single spot. In general, fixations have represented as the duration within every two saccades. The typical duration of fixation set within $100 \mathrm{~ms}$ to $200 \mathrm{~ms}$.

\subsubsection{Blinks}

The Precorneal Tear Film is known to be the front portion of the cornea which has surrounded by a thin fluid that spreads over the region of the cornea through the action of open and close made by the eyelids, namely blinking. Generally, the average rate of blink rests within 12 and 19/min at leisure times. However, there is a number of ecological factors, like brightness, humidity, and temperature, as well as the physical exertion due to mental workload/extreme tiredness that impact the blink rate, where the average blink raises amid 100 and 400 .

\subsection{Optimal feature selection using particle swarm optimization}

Pre-classification process, the feature selection has considered to be a primary phase for eradicating the redundant features, for choosing the parsimonious, information-rich features, and for evading the outfitting at the process of classification. The algorithms of feature transformation and feature selection have widely utilized for diminishing the dimension of feature and choosing valuable features. This study tends to choose the information-rich weighted features by proposing the PSO based feature selection method. In a traditional way, the particles have arbitrarily initialized. Nevertheless, this work utilizes the mixed initialization methodology, in which the initialization of $50 \%$ particles includes a lesser amount of features ( $10 \%$ of overall features), and the rest of the particles utilize a huge amount of features (60\% of overall features).

The evaluation of fitness has viewed as the primary phase in the PSO based feature selection. In general, the design of fitness function significantly includes two standard metrics, i.e. the accuracy and error rate of classification. Nevertheless, such metrics would not be adequate to assess the eminence of the particles while working with the imbalanced dataset, since they delude the efficiency of classification because of the focus on the impact of majority class. So that, this research tends to determine the fitness of each particle by designing the novel fitness function, in which the Geometric mean (G-mean) has exploited to assess the overall performance of the classification.

$$
\begin{aligned}
& \text { Fitness }=\alpha *(1-\text { Gmean })+(1-\alpha) * \\
& \text { ( } \left.\frac{\text { number of selected features }}{\text { All features }}\right)
\end{aligned}
$$

Here, the relevant significance of the classification performance (G-mean) has represented by $\alpha$, whereas the relevant significance of the number of features has indicated by $(1-\alpha)$. According to compare with the number of features, the requirement of classification performance is high, so the value for $\alpha$ defined as 0.8 . The quality of every particle has estimated based on a fitness function. Then, the algorithm upgrades the pbest and gbest subsequently the velocity and position of each particle have updated by the algorithm. Two scenarios decide the update of current pbest and gbest, i.e. i) if the classification performance (G-mean) of the particle's new position was superior to the former pbest and the number of features was lesser than earlier pbest. ii) if the number of features was lesser than former pbest and the classification performance (G-mean) of the new position was identical/better than the current pbest. The updating of the gbest follows the same procedure. The position of a particle signifies the selected feature subset. V-shaped transfer function has enforced inn the suggested binary PSO, in terms of converting the velocity from constant space to probability space, as follows:

$$
\begin{gathered}
S\left(x_{i}^{k}(t)\right)=\frac{2}{\pi} \arctan \left(\frac{\pi}{2} x_{i}^{k}(t)\right) \\
v_{i}^{k}(t)=w * v_{i}^{i}(t)+c_{1} * \text { rand } \\
*\left(\text { pbest }_{i}^{k}(t)-x_{i}^{k}(t)\right)+c_{2} \\
\\
* \operatorname{rand}^{k}\left(\text { gbest }_{i}^{k}(t)-x_{i}^{k}(t)\right)
\end{gathered}
$$


The subsequent rule of position update must be applied, since the v-shaped transfer function has been used.

$$
x_{i}^{k}(t+1)=\left\{\begin{array}{c}
\tilde{x}_{i}^{k}(t) \text { rand }<T\left(v_{i}^{k}(t+1)\right) \\
x_{i}^{k}(t) \text { rand } \geq T\left(v_{i}^{k}(t+1)\right)
\end{array}\right.
$$

The simulation process of PSO stops, if converged with the predetermined stopping condition, such as highest number of iterations/best fitness value, in which the highest number defined for iteration is 100 . On the other hand, if particles have surrounded by local optimum, they will reset to 0 .

Defined the initial value of $w$ as 1.4 and it changes according to the iteration, as expressed by the subsequent equation,

$$
\mathrm{W}=(\mathrm{w}-0.4) *\left(\mathrm{t}_{\max }-\mathrm{t}\right) /\left(\mathrm{t}_{\max }+0.4\right)
$$

here, the highest number of iterations denoted by $t_{\max }$, and the current iteration has notated by $\mathrm{t}$.

\subsection{Detecting epilepsy using thirteen layer convolutional neural network}

The CNN comprises the essential layers, namely the convolution layer, max-pooling layer, and fully connected (dense) layer. Although the network generally inclines for efficient learning, as regards to attaining deeper, yet there is a possibility to impact on the duration of computation. To overcome this issue, we deliberated for developing the network structure that solely needs lesser time for computing. The superior performance has achieved in the process of classification that accompanies the well-regulated parameters in the event of the training phase. The convolutional layer has entwined to the input signal through a kernel (window). Postconvolution, a feature map has produced for the succeeding layer. Subsequently, the batch normalization layer has assigned to stream within the in-between layers, concerning the normalization of input data that facilitates the quicker learning and boosting. Besides, the involvement of rectified linear unit aims at the threshold of input data and diminishes the redundancies among data. Afterwards, the max-pooling layer takes place to decrease the feature map size. In the fully connected layer, each neuron of the max-pooling layer has correlated with one and all, in which the output forecasts the result (Normal/PD) of the input signal.

The implementation of the structural design of a thirteenlayer CNN surpasses the requirement of the conventional feature representation stages. Figure 2 outlines the structure of the proposed approach.

The conventional convolutional neural network was designed with thirteen layers, to match the performance metrics of Genetic based CNN. This process also includes the algorithm called Adam optimization. The activation function applied for all the layers is a piecewise linear function called Rectified Linear Unit (ReLU) function. This function overcomes vanishing gradient problem, allowing the models to learn faster and perform better. The final layer consists of Softmax function, which gives a probability of the label candidates, known as list of classes.

Based on the rendered dataset, the overall parameters are correspondingly regulated, which proves the maximum accuracy of training. The implementation of the structural design of a thirteen-layer $\mathrm{CNN}$ surpasses the requirement of the conventional feature representation stages.
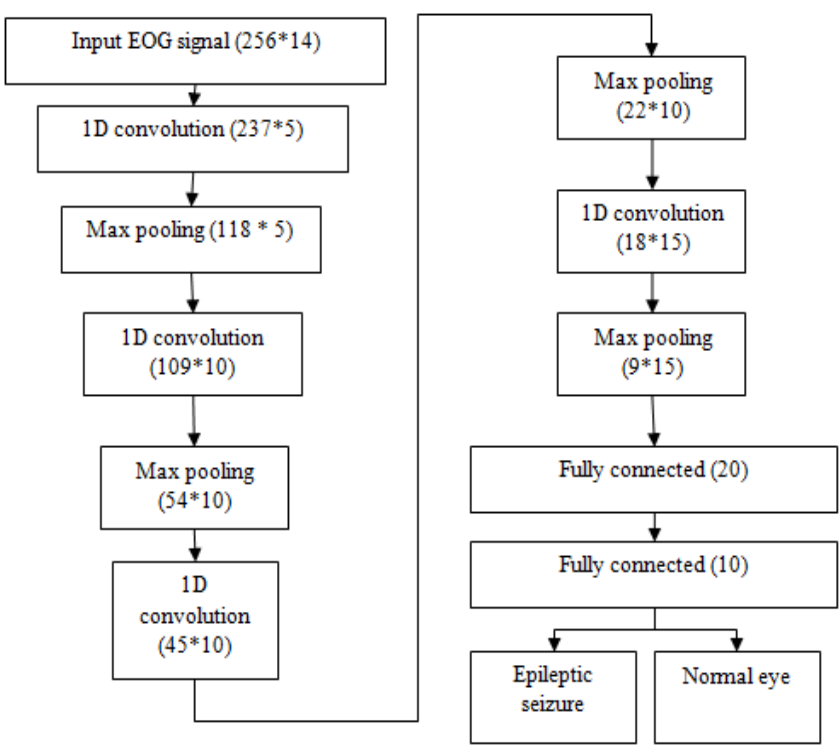

Figure 2. Proposed thirteen layer $\mathrm{CNN}$

The model has processed through two phases in order to authenticate the model, i.e. i) data training, and ii) model testing. i) The overall data has segmented as ten equal portions by presenting the stratified tenfold cross-validation, from which nine have utilized for training, and the remaining one reserved for testing the model. The iteration for ten times ensures the participation of overall portions during the stages of training and testing. ii) $20 \%$ of the cross-validation training data have designated to authenticate the model as regards the evaluation of training improvements at post-completion of each iteration. The process also includes the algorithm called Adam optimization that accompanies the learning rate of 0.0001 , and applied some activation functions, especially the function named ReLU has been applied with entire layers and another function Softmax with the final layer. Subsequently, the dropout layer has been assigned with the dropout value of 0.5 . Based on the rendered dataset, the overall parameters have correspondingly regulated, which proves the maximum accuracy of training. Furthermore, the Brute Force approach helps attaining the kernel size and the number of filters.

\section{OBTAINED RESULTS AND EVALUATIONS}

The proposed thirteen layer is implemented and the outputs are verified in MATLAB simulation tool. The performance metrics are evaluated and measured. This work primarily aims for detecting epileptic seizure using EOG signal. The acquired data set of EOG signals is denoised using the pre-processing techniques.

The Figure 3 represents the signal of normal and epileptic subjects. As the representation itself specifies that epileptic signal has large surface area compared to the normal signal. The variations in the signal amplitudes can also be observed and differentiated in both the categories.

Figure 4 represents the approach of Hybrid Dynamic Time Wrapping based Hidden Markov Model (HDWT-HMM) used for noise reduction and the graph variation of the noise reduction signal. The highest peaks clearly show the variations of the preprocessed signal.

Figure 5 shows the PSO curve of the optimal feature selection process which is done to improvise the classification 
outcome. From the figure it is confirmed that the convergence of the fitness measure reaches after 20 number of iterations from reducing from the convergence rate 0.53 . The receiver operating characteristics curve for classification is shown in Figure 6.

The measured performance metrics used for the evaluation are accuracy, precision, recall and f-measure. These metrics are compared with other existing research works named $\mathrm{CNN}$, GCNN [24] and the proposed algorithm.

Precision: Precision is the ratio of true positive values against true and false positives. It is represented as

$$
\operatorname{Precision}(\mathrm{P})=\frac{T_{p}}{T_{p}+F_{p}}
$$
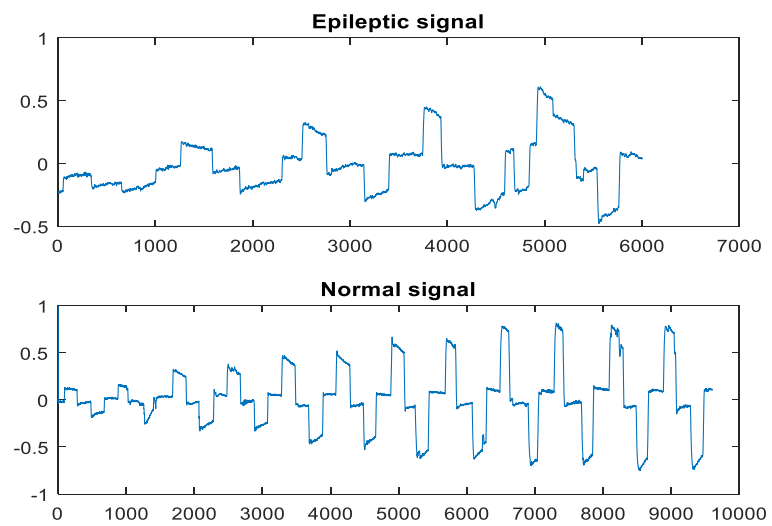

Figure 3. Graph for epileptic signal and the normal signal

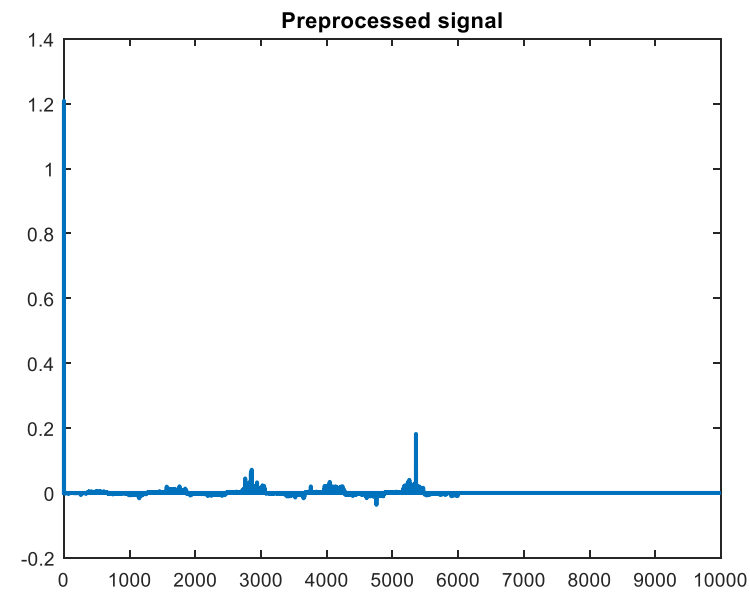

Figure 4. Preprocessed signal graph

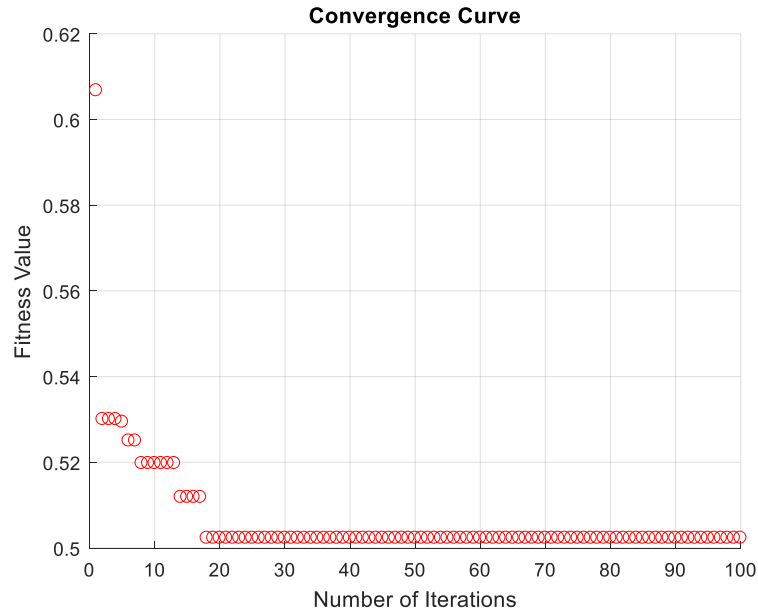

Figure 5. PSO curve

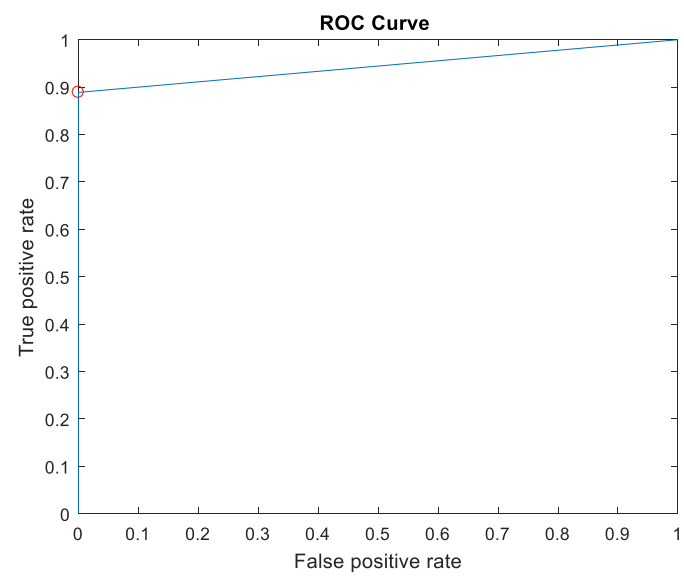

Figure 6. Receiver operating characteristics curve comparison

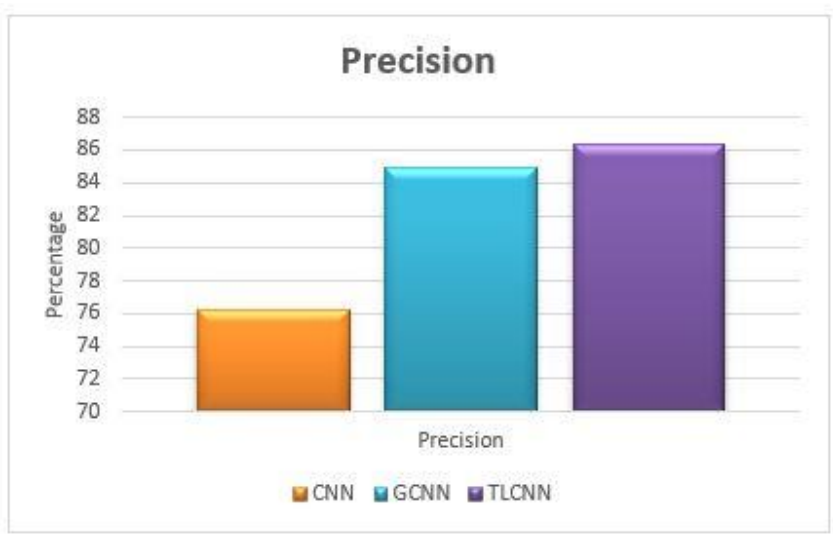

Figure 7. Precision comparison

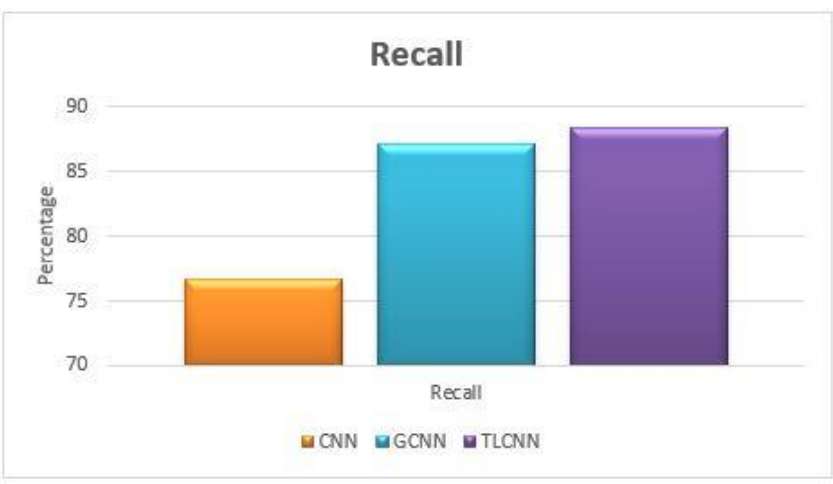

Figure 8. Recall comparison

Figure 7 depicts the results of existing and proposed method in terms of precision.

Recall: Recall is the ratio of true positives against true positive and false negative.

$$
\operatorname{Recall}(\mathrm{R})=\frac{T_{p}}{T_{p}+F_{n}}
$$

From the above chart, Figure 8 represents that the suggested approach of TLCNN procures optimum recall. Thus, proposed algorithm of TLCNN is superior than previous work ESDGCNN and existing algorithm of CNN.

F-measure: It is nothing but the trial accurate assessment. The score is calculated on the basis of precision $\mathrm{p}$ and the recall $r$ of the trial. 


$$
\text { F-measure }=2 \cdot \frac{P \cdot R}{P+R}
$$

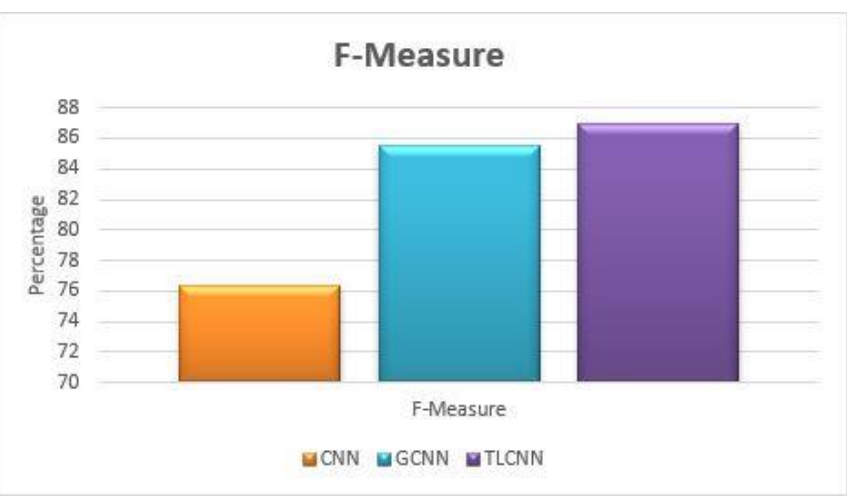

Figure 9. F-measure comparison

From the above chart, Figure 9 demonstrates that the suggested approach of NESD-TLCNN offers greater fmeasure. Thus, the proposed algorithm of TLCNN is superior to the previous work GCNN and the existing algorithm of CNN [25-27].

Accuracy: The performance of the system is judged by the value of accuracy. It is defined to be the ratio of sum of true positives and true negatives against the sum of classification parameters [28-30]. The expression of accuracy is as follows

$$
\text { Accuracy }=\frac{T_{p}+T_{n}}{\left(T_{p}+T_{n}+F_{p}+F_{n}\right)}
$$

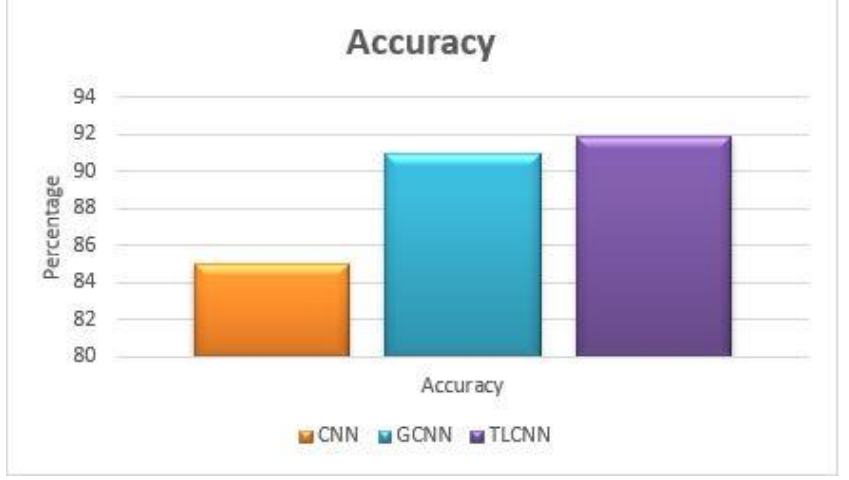

Figure 10. Comparison of accuracy metrics

From the above chart, Figure 10 represents that the suggested approach of TLCNN offers optimal accuracy than other approaches.

Percentage values of the performance metrics of the existing and proposed classification algorithms, convolutional neural network (CNN), genetic convolutional neural network (GCNN) and thirteen-layer convolutional neural network (TLCNN) are presented in Table 1.

Table 1. Implemented algorithms comparison of

\begin{tabular}{|c|c|c|c|}
\hline \multirow[b]{2}{*}{ Metrics } & \multicolumn{3}{|c|}{ Results in \% for various classifier algorithms } \\
\hline & CNN & GCNN & TLCNN \\
\hline Recall & 76.72 & 87.06 & 88.36 \\
\hline Specificity & 88.9 & 92.8 & 93.5 \\
\hline Accuracy & 85.04 & 90.98 & 91.87 \\
\hline Precision & 76.23 & 84.87 & 86.31 \\
\hline F-Measure & 76.34 & 85.47 & 86.98 \\
\hline
\end{tabular}
performance Metrics in (\%)
The graphical representation of comparison performance metrics of implemented classification algorithms is shown in Figure 11

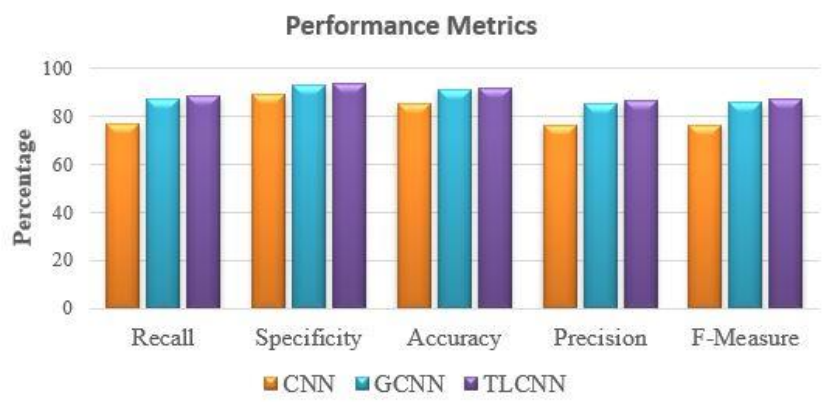

Figure 11. Graphical representation performance metrics of implemented classification algorithms

\section{CONCLUSION}

An early stage of this study has involved the method, called Hybrid Dynamic Time Wrapping based Hidden Markov Model (HDWT-HMM) to carry out the identification and elimination of Background Noise, in which the Noise has evaluated on the basis of distance parameter. Post-evaluation of Noise, the accuracy in identifying the epileptic seizure has assured through executing the Feature Extraction process. This research has taken the features into account, such as Fixation, Blink Features, and Saccade. Subsequently, the Optimal Feature Selection has implemented through the strategy, namely Particle Swarm Optimization (PSO). In addition, the system of Thirteen Layer Convolution Neural Network (TLCNN) has employed to learn and contrast the epileptic seizure from normal eyes. Moreover, this research study has completely scrutinized under the simulation environment of MATLAB so as to establish that the proposed approach has significantly intended for the optimum result than other research study.

\section{REFERENCES}

[1] Arunkumar, N., Kumar, K.R., Venkataraman, V. (2016). Automatic detection of epileptic seizures using new entropy measures. Journal of Medical Imaging and Health Informatics, 6(3): 724-730. https://doi.org/10.1166/jmihi.2016.1736

[2] Tzimourta, K.D., Tzallas, A.T., Giannakeas, N., Astrakas, L.G., Tsalikakis, D.G., Tsipouras, M.G. (2017). Epileptic seizures classification based on long-term EEG signal wavelet analysis. In International Conference on Biomedical and Health Informatics, 66: 165-169. https://doi.org/10.1007/978-981-10-7419-6_28

[3] Ryvlin, P., Rheims, S., Lhatoo, S.D. (2019). Risks and predictive biomarkers of sudden unexpected death in epilepsy patient. Current Opinion in Neurology, 32(2): 205-212. https://doi.org/10.1097/WCO.0000000000000668

[4] Myers, K.A., Bello-Espinosa, L.E., Symonds, J.D., Zuberi, S.M., Clegg, R., Sadleir, L.G., Scheffer, I.E. (2018). Heart rate variability in epilepsy: A potential biomarker of sudden unexpected death in epilepsy risk. Epilepsia, 59(7): 
https://doi.org/10.1111/epi.14438

[5] Maddirala, A.K., Shaik, R.A. (2017). Separation of sources from single-channel EEG signals using independent component analysis. IEEE Transactions on Instrumentation and Measurement, 67(2): 382-393. https://doi.org/10.1109/TIM.2017.2775358

[6] Arnin, J., Kahani, D., Lakany, H., Conway, B.A. (2018). Evaluation of different signal processing methods in time and frequency domain for brain-computer interface applications. In 2018 40th Annual International Conference of the IEEE Engineering in Medicine and Biology Society (EMBC), pp. 235-238. https://doi.org/10.1109/EMBC.2018.8512193

[7] Geethu, V., Santhoshkumar, S. (2018). An efficient FPGA realization of seizure detection from EEG signal using wavelet transform and statistical features. IETE Journal of Research, 66(3): 1-11. https://doi.org/10.1080/03772063.2018.1491806

[8] Noor, N.M.M., bin Kamarudin, M.Q. (2016). Study the different level of eye movement based on electrooculography (EOG) technique. In 2016 IEEE EMBS Conference on Biomedical Engineering and Sciences (IECBES), pp. 792-796. https://doi.org/10.1109/IECBES.2016.7843559

[9] Shimizu, J., Chernyshov, G. (2016). Eye movement interactions in google cardboard using a low cost EOG setup. In Proceedings of the 2016 ACM International Joint Conference on Pervasive and Ubiquitous Computing: $\quad$ Adjunct, pp. 1773-1776. https://doi.org/10.1145/2968219.2968274

[10] Gao, B., He, Z., He, B., Gu, Z. (2019). Wearable eye health monitoring sensors based on peacock tail-inspired inverse opal carbon. Sensors and Actuators B: Chemical, 288: 734-741. https://doi.org/10.1016/j.snb.2019.03.029

[11] Thodoroff, P., Pineau, J., Lim, A. (2016). Learning robust features using deep learning for automatic seizure detection. In Machine Learning for Healthcare Conference, pp. 178-190.

[12] Hong, K.S., Khan, M.J., Hong, M.J. (2018). Feature extraction and classification methods for hybrid fNIRSEEG brain-computer interfaces. Frontiers in Human Neuroscience, 12 : 246. https://doi.org/10.3389/fnhum.2018.00246

[13] Giraldo-Suarez, E., Martínez-Vargas, J.D., CastellanosDominguez, G. (2016). Reconstruction of neural activity from EEG data using dynamic spatiotemporal constraints. International Journal of Neural Systems, 26(07): 1650026. https://doi.org/10.1142/S012906571650026X

[14] Xiahou, J., He, H., Wei, K., She, Y. (2016). Integrated approach of dynamic human eye movement recognition and tracking in real time. In 2016 International Conference on Virtual Reality and Visualization (ICVRV), pp. 94-101. https://doi.org/10.1109/ICVRV.2016.23

[15] Kubacki, A., Jakubowski, A. (2018). Controlling the industrial robot model with the hybrid BCI based on EOG and eye tracking. In AIP Conference Proceedings 2029(1): 020032. https://doi.org/10.1063/1.5066494

[16] Cafasso, A., Karlsson, S. (2017). Automatic detection of saccadic eye movements using EOG for analysing effects of cognitive distraction during driving. Master's thesis. https://hdl.handle.net/20.500.12380/250503

[17] Bin Suhaimi, M.S.A., Matsushita, K., Sasaki, M., Njeri, W. (2019). 24-gaze-point calibration method for improving the precision of AC-EOG gaze estimation. Sensors, $19(17)$ : 3650. https://doi.org/10.3390/s19173650

[18] Baba, R., Sugi, T., Matsuda, Y., Goto, S., Yamasaki, T. (2019). Extraction of eye movements for a communication system by EOG signal using DC amplifier. In Proceedings of the ISCIE International Symposium on Stochastic Systems Theory and its Applications, 2019: https://doi.org/10.5687/sss.2019.183

[19] Roy, S., De, A., Panigrahi, N. (2019). Saccade and Fix Detection from EOG Signal. In 2019 IEEE International Symposium on Smart Electronic Systems (iSES)(Formerly iNiS), pp. 406-408. https://doi.org/10.1109/iSES47678.2019.00099

[20] Kumar, D., Sharma, A. (2016). Electrooculogram-based virtual reality game control using blink detection and gaze calibration. In 2016 International Conference on Advances in Computing, Communications and Informatics (ICACCI), pp. 2358-2362. https://doi.org/10.1109/ICACCI.2016.7732407

[21] Sharma, K., Jain, N., Pal, P.K. (2020). Detection of eye closing/opening from EOG and its application in robotic arm control. Biocybernetics and Biomedical Engineering, 40(1): 173-186. https://doi.org/10.1016/j.bbe.2019.10.004

[22] Schmidt, J., Laarousi, R., Stolzmann, W., Karrer-Gauß, K. (2018). Eye blink detection for different driver states in conditionally automated driving and manual driving using EOG and a driver camera. Behavior Research Methods, 50(3): 1088-1101. https://doi.org/10.3758/s13428-017-0928-0

[23] Ryu, J., Lee, M., Kim, D.H. (2019). EOG-based eye tracking protocol using baseline drift removal algorithm for long-term eye movement detection. Expert Systems with Applications, 131: 275-287. https://doi.org/10.1016/j.eswa.2019.04.039

[24] Bulling, A., Gellersen, H. (2010). Toward mobile eyebased human-computer interaction. IEEE Pervasive Computing, 9(4): 8-12. https://doi.org/10.1109/MPRV.2010.86

[25] Talukder, M., Aktar, A., Neelima, A., Rahman, A. (2018). EOG based home automation system by cursor movement using a graphical user interface (GUI). In 2018 IEEE International WIE Conference on Electrical and Computer Engineering (WIECON-ECE), pp. 1-4. https://doi.org/10.1109/WIECON-ECE.2018.8783025

[26] Priyanka Brahmaiah, V., Padma Sai, Y., Giri Prasad, M.N. (2018). Performance and analysis of EOG signal through eye movements. Journal of Advanced Research in Dynamical \& Control Systems, 10: 2039-2045.

[27] Kumar, D., Sharma, A. (2016). Electrooculogram-based virtual reality game control using blink detection and gaze calibration. In 2016 International Conference on Advances in Computing, Communications and Informatics (ICACCI), pp. 2358-2362. https://doi.org/10.1109/ICACCI.2016.7732407

[28] Belo, D., Rodrigues, J., Vaz, J.R., Pezarat-Correia, P., Gamboa, H. (2017). Biosignals learning and synthesis using deep neural networks. Biomedical Engineering Online, 16(1): 1-17. https://doi.org/10.1186/s12938-0170405-0.

[29] Latifoğlu, F., Esas, M.Y., Demirci, E. (2020). Diagnosis of attention-deficit hyperactivity disorder using EOG 
signals: a new approach. Biomedical Engineering/Biomedizinische Technik, 65(2): 149-164. https://doi.org/10.1515/bmt-2019-0027

[30] Christensen, J.A., Koch, H., Frandsen, R., Kempfner, J., Arvastson, L., Christensen, S.R., Jennum, P. (2013) Classification of iRBD and Parkinson's disease patients based on eye movements during sleep. In 2013 35th

Annual International Conference of the IEEE

Engineering in Medicine and Biology Society (EMBC)

pp.

https://doi.org/10.1109/EMBC.2013.6609531 